EPJ Web of Conferences 107, 05007 (2016)

DOI: 10.1051/epjconf/201610705007

(C) Owned by the authors, published by EDP Sciences, 2016

\title{
Nuclear orbital and spin scissors with pairing
}

\author{
Evgeny Balbutsev ${ }^{1, a}$ and Irina Molodtsova ${ }^{1}$ \\ ${ }^{1}$ Joint Institute for Nuclear Research, 141980 Dubna, Moscow region, Russia
}

\begin{abstract}
Nuclear scissors modes are considered in the frame of the Wigner function moments method generalized to take into account spin degrees of freedom and pair correlations simultaneously. A new source of nuclear magnetism, connected with counter-rotation of spins up and down around the symmetry axis (hidden angular momenta), is discovered. Its inclusion into the theory allows one to improve substantially the agreement with experimental data in the description of energies and transition probabilities of scissors modes in rare earth nuclei.
\end{abstract}

\section{Introduction}

The nuclear scissors mode was predicted by R. R. Hilton $[1,2]$, as a counter-rotation of protons against neutrons in deformed nuclei. First successful estimation of its energy in the frame of RPA was done by T. Suzuki and D. J. Rowe [3]. First phenomenological model of this phenomenon was suggested by N. Lo Iudice and F. Palumbo [4]. Its experimental discovery [5] has initiated a cascade of theoretical studies. An excellent review of their twenty years development was given by D. Zawischa [6]. The most successful in the description of the energy and excitation probability of the scissors mode turned out the sum rule method [7] and RPA [8]. Very interesting qualitative features of the scissors mode were discovered with the help of Wigner Function Moments (WFM) method [912]. This method is a purely microscopic one, because it is based on the Time Dependent Hartree-Fock (TDHF) equation. In recent papers $[10,11]$ the WFM method was applied for the first time to solve the TDHF equations including spin dynamics. The most remarkable result was the discovery of a new type of nuclear collective motion: rotational oscillations of "spin-up" nucleons with respect of "spin-down" nucleons (the spin scissors mode). It turns out that the experimentally observed group of peaks in the energy interval $2-4 \mathrm{MeV}$ corresponds very likely to two different types of motion: the orbital scissors mode and this new mode, namely, the spin scissors one. The pictorial view of these two intermingled scissors is shown in Fig. 1. In the present work we extend the WFM method to take into account spin degrees of freedom and pair correlations simultaneously.

\section{Wigner transformation of TDHFB equations}

The Time-Dependent Hartree-Fock-Bogoliubov (TDHFB) equations in matrix formulation are the following [13]:

\footnotetext{
ae-mail: balbuts@theor.jinr.ru
}

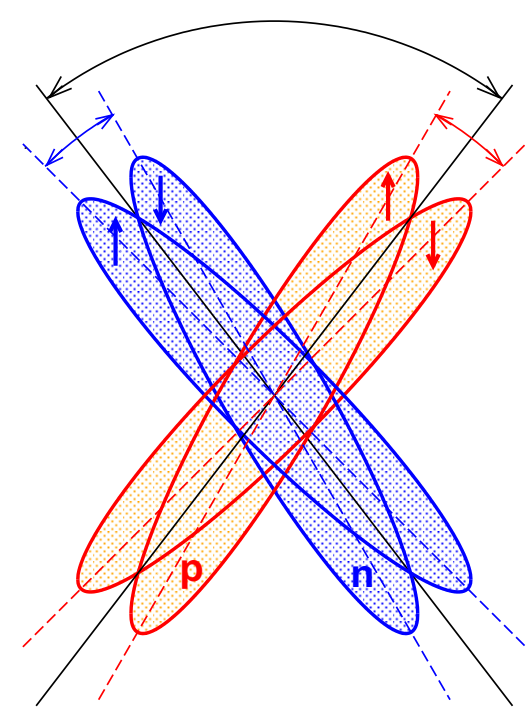

Figure 1. (Color online) Pictorial representation of two intermingled scissors: the orbital (neutrons versus protons) scissors + spin (spin-up nucleons versus spin-down nucleons) scissors. Arrows inside of ellipses show the direction of spin projections, $\mathbf{p}$ protons, $\mathbf{n}$ - neutrons.

$$
\begin{gathered}
i \hbar \dot{\mathcal{R}}=[\mathcal{H}, \mathcal{R}] \\
\text { with } \mathcal{R}=\left(\begin{array}{cc}
\hat{\rho} & -\hat{\kappa} \\
-\hat{\kappa}^{\dagger} & 1-\hat{\rho}^{*}
\end{array}\right), \quad \mathcal{H}=\left(\begin{array}{cc}
\hat{h} & \hat{\Delta} \\
\hat{\Delta}^{\dagger} & -\hat{h}^{*}
\end{array}\right),
\end{gathered}
$$

where $\hat{\rho}$ is the normal density matrix, $\hat{h}$ is the Hamiltonian, $\hat{\kappa}$ is the anomalous density and $\hat{\Delta}-$ the pairing gap. The detailed form of the TDHFB equations is

$$
\begin{aligned}
& i \hbar \dot{\hat{\rho}}=\hat{h} \hat{\rho}-\hat{\rho} \hat{h}-\hat{\Delta} \hat{\kappa}^{\dagger}+\hat{\kappa} \hat{\Delta}^{\dagger}, \\
& i \hbar \hat{\kappa}=-\hat{h} \hat{\kappa}-\hat{\kappa} \hat{h}^{*}+\hat{\Delta}-\hat{\Delta} \hat{\rho}^{*}-\hat{\rho} \hat{\Delta}
\end{aligned}
$$

plus the pair of complex conjugated equations. Let us consider their matrix form in coordinate space keeping all spin 
indices $s, s^{\prime}, s^{\prime \prime}$ :

$$
\begin{aligned}
& i \hbar\left\langle\mathbf{r}, s|\dot{\hat{\rho}}| \mathbf{r}^{\prime \prime}, s^{\prime \prime}\right\rangle=\sum_{s^{\prime}} \int d^{3} r^{\prime}\left(\left\langle\mathbf{r}, s|\hat{h}| \mathbf{r}^{\prime}, s^{\prime}\right\rangle\left\langle\mathbf{r}^{\prime}, s^{\prime}|\hat{\rho}| \mathbf{r}^{\prime \prime}, s^{\prime \prime}\right\rangle\right. \\
& -\left\langle\mathbf{r}, s|\hat{\rho}| \mathbf{r}^{\prime}, s^{\prime}\right\rangle\left\langle\mathbf{r}^{\prime}, s^{\prime}|\hat{h}| \mathbf{r}^{\prime \prime}, s^{\prime \prime}\right\rangle-\left\langle\mathbf{r}, s|\hat{\Delta}| \mathbf{r}^{\prime}, s^{\prime}\right\rangle\left\langle\mathbf{r}^{\prime}, s^{\prime}\left|\hat{\kappa}^{\dagger}\right| \mathbf{r}^{\prime \prime}, s^{\prime \prime}\right\rangle \\
& \left.+\left\langle\mathbf{r}, s|\hat{\kappa}| \mathbf{r}^{\prime}, s^{\prime}\right\rangle\left\langle\mathbf{r}^{\prime}, s^{\prime}\left|\hat{\Delta}^{\dagger}\right| \mathbf{r}^{\prime \prime}, s^{\prime \prime}\right\rangle\right), \\
& i \hbar\left\langle\mathbf{r}, s|\dot{\hat{\kappa}}| \mathbf{r}^{\prime \prime}, s^{\prime \prime}\right\rangle=-\sum_{s^{\prime}} \int d^{3} r^{\prime}\left(\left\langle\mathbf{r}, s|\hat{h}| \mathbf{r}^{\prime}, s^{\prime}\right\rangle\left\langle\mathbf{r}^{\prime}, s^{\prime}|\hat{\kappa}| \mathbf{r}^{\prime \prime}, s^{\prime \prime}\right\rangle\right. \\
& +\left\langle\mathbf{r}, s|\hat{\kappa}| \mathbf{r}^{\prime}, s^{\prime}\right\rangle\left\langle\mathbf{r}^{\prime}, s^{\prime}\left|\hat{h}^{*}\right| \mathbf{r}^{\prime \prime}, s^{\prime \prime}\right\rangle+\left\langle\mathbf{r}, s|\hat{\Delta}| \mathbf{r}^{\prime}, s^{\prime}\right\rangle\left\langle\mathbf{r}^{\prime}, s^{\prime}\left|\hat{\rho}^{*}\right| \mathbf{r}^{\prime \prime}, s^{\prime \prime}\right\rangle \\
& \left.+\left\langle\mathbf{r}, s|\hat{\rho}| \mathbf{r}^{\prime}, s^{\prime}\right\rangle\left\langle\mathbf{r}^{\prime}, s^{\prime}|\hat{\Delta}| \mathbf{r}^{\prime \prime}, s^{\prime \prime}\right\rangle\right)+\left\langle\mathbf{r}, s|\hat{\Delta}| \mathbf{r}^{\prime \prime}, s^{\prime \prime}\right\rangle
\end{aligned}
$$

plus the pair of complex conjugated equations.

It is convenient to work with the Wigner (Fourier) transform $f^{s s^{\prime}}(\mathbf{r}, \mathbf{p}, t)$ of the normal density matrix $\left\langle\mathbf{r}^{\prime}, s^{\prime}|\hat{\rho}| \mathbf{r}^{\prime \prime}, s^{\prime \prime}\right\rangle$ and the Wigner transform $\kappa^{s s^{\prime}}(\mathbf{r}, \mathbf{p}, t)$ of the anomalous density matrix $\left\langle\mathbf{r}^{\prime}, s^{\prime}|\hat{k}| \mathbf{r}^{\prime \prime}, s^{\prime \prime}\right\rangle$. The Wigner transform of (5) is solved by the method of moments in a small amplitude approximation. To this end all functions $f^{s s^{\prime}}(\mathbf{r}, \mathbf{p}, t)$ and $\kappa^{s s^{\prime}}(\mathbf{r}, \mathbf{p}, t)$ are divided into equilibrium part and deviation (variation)

$$
\begin{aligned}
& f^{s s^{\prime}}(\mathbf{r}, \mathbf{p}, t)=f^{s s^{\prime}}(\mathbf{r}, \mathbf{p})_{\mathrm{eq}}+\delta f^{s s^{\prime}}(\mathbf{r}, \mathbf{p}, t), \\
& \kappa^{s s^{\prime}}(\mathbf{r}, \mathbf{p}, t)=\kappa^{s s^{\prime}}(\mathbf{r}, \mathbf{p})_{\mathrm{eq}}+\delta \kappa^{s s^{\prime}}(\mathbf{r}, \mathbf{p}, t) .
\end{aligned}
$$

Then equations are linearized neglecting the terms quadratic in variations.

\section{Model Hamiltonian}

The microscopic Hamiltonian of the model consisting of the harmonic oscillator with spin-orbit potential and separable quadrupole-quadrupole and spin-spin residual interactions is given by

$$
H=\sum_{i=1}^{A}\left[\frac{\hat{\mathbf{p}}_{i}^{2}}{2 m}+\frac{1}{2} m \omega^{2} \mathbf{r}_{i}^{2}-\eta \hat{\mathbf{l}} \hat{\mathbf{S}}_{i}\right]+H_{q q}+H_{s s},
$$

with

$$
\begin{array}{r}
H_{q q}=\sum_{\mu=-2}^{2}(-1)^{\mu}\left\{\bar{\kappa} \sum_{i}^{Z} \sum_{j}^{N}+\frac{\kappa}{2}\left[\sum_{i, j(i \neq j)}^{Z}+\sum_{i, j(i \neq j)}^{N}\right]\right\} \times \\
H_{s S}=\sum_{\mu=-1}^{1}(-1)^{\mu}\left\{\bar{\chi} \sum_{i}^{Z} \sum_{j}^{N}+\frac{\chi}{2}\left[\sum_{i, j(i \neq j)}^{Z}+\sum_{i, j(i \neq j)}^{N}\right]\right\} \times \\
\hat{S}_{-\mu}(i) \hat{S}_{\mu}(j) \delta\left(\mathbf{r}_{i}-\mathbf{r}_{j}\right),
\end{array}
$$

where $N$ and $Z$ are the numbers of neutrons and protons, $A=N+Z, q_{2 \mu}(\mathbf{r})=\sqrt{16 \pi / 5} r^{2} Y_{2 \mu}(\theta, \phi)-$ quadrupole operator and $\hat{S}_{\mu}$ are spin matrices [14].

\subsection{Mean Field}

Let us analyze the mean field generated by this Hamiltonian.

\subsubsection{Spin-orbital Potential}

Written in cyclic coordinates, the spin-orbit part of the Hamiltonian reads

$$
\hat{h}_{l s}=-\eta \sum_{\mu=-1}^{1}(-)^{\mu} \hat{l}_{\mu} \hat{S}_{-\mu}=-\eta\left(\begin{array}{cc}
\hat{l}_{0} \frac{\hbar}{2} & \hat{l}_{-1} \frac{\hbar}{\sqrt{2}} \\
-\hat{l}_{1} \frac{\hbar}{\sqrt{2}} & -\hat{l}_{0} \frac{\hbar}{2}
\end{array}\right),
$$

where

$$
\hat{l}_{\mu}=-\hbar \sqrt{2} \sum_{\nu, \alpha} C_{1 v, 1 \alpha}^{1 \mu} r_{\nu} \nabla_{\alpha}
$$

$r_{-1}, r_{0}, r_{1}-$ cyclic coordinates, $C_{1 \sigma, 1 v}^{\lambda \mu}$ is a Clebsch-Gordan coefficient, and

$$
\begin{aligned}
& \hat{l}_{1}=\hbar\left(r_{0} \nabla_{1}-r_{1} \nabla_{0}\right)=-\frac{1}{\sqrt{2}}\left(\hat{l}_{x}+i \hat{l}_{y}\right), \\
& \hat{l}_{0}=\hbar\left(r_{-1} \nabla_{1}-r_{1} \nabla_{-1}\right)=\hat{l}_{z}, \\
& \hat{l}_{-1}=\hbar\left(r_{-1} \nabla_{0}-r_{0} \nabla_{-1}\right)=\frac{1}{\sqrt{2}}\left(\hat{l}_{x}-i \hat{l}_{y}\right), \\
& \hat{l}_{x}=-i \hbar\left(y \nabla_{z}-z \nabla_{y}\right), \quad \hat{l}_{y}=-i \hbar\left(z \nabla_{x}-x \nabla_{z}\right), \\
& \hat{l}_{z}=-i \hbar\left(x \nabla_{y}-y \nabla_{x}\right) .
\end{aligned}
$$

Matrix elements of $\hat{h}_{l s}$ in coordinate space can obviously be written [10] as

$$
\begin{array}{r}
\left\langle\mathbf{r}_{1}, s_{1}\left|\hat{h}_{l s}\right| \mathbf{r}_{2}, s_{2}\right\rangle=-\frac{\hbar}{2} \eta\left[\hat{l}_{0}\left(\mathbf{r}_{1}\right)\left(\delta_{s_{1} \uparrow} \delta_{s_{2} \uparrow}-\delta_{s_{1} \downarrow} \delta_{s_{2} \downarrow}\right)\right. \\
\left.+\sqrt{2} \hat{l}_{-1}\left(\mathbf{r}_{1}\right) \delta_{s_{1} \uparrow} \delta_{s_{2} \downarrow}-\sqrt{2} \hat{l}_{1}\left(\mathbf{r}_{1}\right) \delta_{s_{1} \downarrow} \delta_{s_{2} \uparrow}\right] \delta\left(\mathbf{r}_{1}-\mathbf{r}_{2}\right) .
\end{array}
$$

Their Wigner transform reads [10]

$$
\begin{aligned}
& h_{l s}^{s_{1} s_{2}}(\mathbf{r}, \mathbf{p})=-\frac{\hbar}{2} \eta\left[l_{0}(\mathbf{r}, \mathbf{p})\left(\delta_{s_{1} \uparrow} \delta_{s_{2} \uparrow}-\delta_{s_{1} \downarrow} \delta_{s_{2} \downarrow}\right)\right. \\
& \left.+\sqrt{2} l_{-1}(\mathbf{r}, \mathbf{p}) \delta_{s_{1} \uparrow} \delta_{s_{2} \downarrow}-\sqrt{2} l_{1}(\mathbf{r}, \mathbf{p}) \delta_{s_{1} \downarrow} \delta_{s_{2} \uparrow}\right]
\end{aligned}
$$

where $l_{\mu}=-i \sqrt{2} \sum_{v, \alpha} C_{1 v, 1 \alpha}^{1 \mu} r_{\nu} p_{\alpha}$.

\subsubsection{Quadrupole-quadrupole interaction}

The contribution of $H_{q q}$ to the mean field potential is easily found by replacing one of the $q_{2 \mu}$ operators by the average value. We have

$$
V_{q q}^{\tau}=\sqrt{6} \sum_{\mu}(-1)^{\mu} Z_{2-\mu}^{\tau+} q_{2 \mu}
$$

Here

$$
\begin{aligned}
& Z_{2 \mu}^{n+}=\kappa R_{2 \mu}^{n+}+\bar{\kappa} R_{2 \mu}^{p+}, \quad Z_{2 \mu}^{p+}=\kappa R_{2 \mu}^{p+}+\bar{\kappa} R_{2 \mu}^{n+}, \\
& R_{2 \mu}^{\tau+}(t)=\frac{1}{\sqrt{6}} \int d(\mathbf{p}, \mathbf{r}) q_{2 \mu}(\mathbf{r}) f^{\tau+}(\mathbf{r}, \mathbf{p}, t)
\end{aligned}
$$

with $\int d(\mathbf{p}, \mathbf{r}) \equiv(2 \pi \hbar)^{-3} \int d^{3} p \int d^{3} r, \tau$ being the isospin index and $f^{+}=f^{\uparrow \uparrow}+f^{\downarrow \downarrow}$. For the isoscalar strength constant $\kappa_{0}=\frac{1}{2}(\kappa+\bar{\kappa})$ we take the self-consistent value [15] $\kappa_{0}=-m \bar{\omega}^{2} /\left(4 Q_{00}\right)$ with $Q_{00}=\frac{3}{5} A R^{2}, \bar{\omega}^{2}=\omega_{0}^{2} /[(1+$ $\left.\left.\frac{4}{3} \delta\right)^{2 / 3}\left(1-\frac{2}{3} \delta\right)^{1 / 3}\right], \hbar \omega_{0}=41 / A^{1 / 3} \mathrm{MeV}$. The isovector strength constant $\kappa_{1}=\frac{1}{2}(\kappa-\bar{\kappa})$ is determined by the relation $\kappa_{1}=\alpha \kappa_{0}$. We take $\alpha=-2$ [16]. 


\subsubsection{Spin-spin interaction}

The analogous expression for $H_{s s}$ is found in a standard way [11] with the following result for the Wigner transform of the proton mean field

$$
\begin{aligned}
V_{p}^{s s^{\prime}}(\mathbf{r}, t)= & 3 \chi \frac{\hbar^{2}}{8}\left[\delta_{s \downarrow} \delta_{s^{\prime} \uparrow} n_{p}^{\downarrow \uparrow}+\delta_{s \uparrow} \delta_{s^{\prime} \downarrow} n_{p}^{\uparrow \downarrow}\right. \\
& \left.-\delta_{s \downarrow} \delta_{s^{\prime} \downarrow} n_{p}^{\uparrow \uparrow}-\delta_{s \uparrow} \delta_{s^{\prime} \uparrow} n_{p}^{\downarrow \downarrow}\right] \\
+ & \bar{\chi} \frac{\hbar^{2}}{8}\left[2 \delta_{s \downarrow} \delta_{s^{\prime} \uparrow} n_{n}^{\downarrow \uparrow}+2 \delta_{s \uparrow} \delta_{s^{\prime} \downarrow} n_{n}^{\uparrow \downarrow}\right. \\
+ & \left.\left(\delta_{s \uparrow} \delta_{s^{\prime} \uparrow}-\delta_{s \downarrow} \delta_{s^{\prime} \downarrow}\right)\left(n_{n}^{\uparrow \uparrow}-n_{n}^{\downarrow \downarrow}\right)\right],
\end{aligned}
$$

where $n_{\tau}^{s s^{\prime}}(\mathbf{r}, t)=\int \frac{d^{3} p}{(2 \pi \hbar)^{3}} f_{\tau}^{s s^{\prime}}(\mathbf{r}, \mathbf{p}, t)$. The Wigner transform of the neutron mean field $V_{n}^{s s^{\prime}}$ is obtained from (15) by the obvious change of indices $p \leftrightarrow n$. The values of strength constants $\chi$ and $\bar{\chi}$ are taken from [17], where the notation $\chi=K_{s} / A, \bar{\chi}=q \chi$ was introduced. The constants were extracted by the authors of [17] from Skyrme forces following the standard procedure, the residual interaction being defined in terms of the second derivatives of the Hamiltonian density $H(\rho)$ with respect to the one-body densities $\rho$. Different variants of Skyrme forces produce different strength constants of spin-spin interaction. The most consistent results were obtained with SG1, SG2 and Sk3 forces. It was found in [17] that the best agreement with experimental data in the description of spin-flip resonances is obtained with the slightly modified values of $K_{s}$ and $q$ extracted from SG2 forces: $K_{s}=50, q=-1$. Thus, we use these numbers.

\subsection{Pair potential}

The Wigner transform of the pair potential (pairing gap) $\Delta(\mathbf{r}, \mathbf{p})$ is related to the Wigner transform of the anomalous density by

$$
\Delta(\mathbf{r}, \mathbf{p})=-\int \frac{\mathrm{d} \mathbf{p}^{\prime}}{(2 \pi \hbar)^{3}} v\left(\left|\mathbf{p}-\mathbf{p}^{\prime}\right|\right) \kappa\left(\mathbf{r}, \mathbf{p}^{\prime}\right),
$$

where $v(p)$ is a Fourier transform of the two-body interaction. We take for the pairing interaction a simple Gaussian form with the strength $V_{0}=25 \mathrm{MeV}$ and range $r_{p}=1.9 \mathrm{fm}[13] v(p)=\beta \mathrm{e}^{-\alpha p^{2}}$ with $\beta=-\left|V_{0}\right|\left(r_{p} \sqrt{\pi}\right)^{3}$ and $\alpha=r_{p}^{2} / 4 \hbar^{2}$.

\section{Collective variables and equations of motion}

Integrating the Wigner transform of the set of Eqs. (5) over phase space with the weights $W=\{r \otimes p\}_{\lambda \mu},\{r \otimes r\}_{\lambda \mu},\{p \otimes p\}_{\lambda \mu}, \quad$ and 1 one gets dynamic equations for the following collective variables:

$$
\begin{aligned}
& \mathcal{L}_{\lambda \mu}^{\tau S}(t)=\int d(\mathbf{p}, \mathbf{r})\{r \otimes p\}_{\lambda \mu} \delta f_{o}^{\tau \zeta}(\mathbf{r}, \mathbf{p}, t), \\
& \mathcal{R}_{\lambda \mu}^{\tau S}(t)=\int d(\mathbf{p}, \mathbf{r})\{r \otimes r\}_{\lambda \mu} \delta f_{e}^{\tau S}(\mathbf{r}, \mathbf{p}, t), \\
& \mathcal{P}_{\lambda \mu}^{\tau S}(t)=\int d(\mathbf{p}, \mathbf{r})\{p \otimes p\}_{\lambda \mu} \delta f_{e}^{\tau \zeta}(\mathbf{r}, \mathbf{p}, t),
\end{aligned}
$$

$$
\begin{aligned}
& \mathcal{F}^{\tau \varsigma}(t)=\int d(\mathbf{p}, \mathbf{r}) \delta f_{e}^{\tau \varsigma}(\mathbf{r}, \mathbf{p}, t), \\
& \tilde{\mathcal{L}}_{\lambda \mu}^{\tau}(t)=\int d(\mathbf{p}, \mathbf{r})\{r \otimes p\}_{\lambda \mu} \delta \kappa_{o}^{\tau i}(\mathbf{r}, \mathbf{p}, t), \\
& \tilde{\mathcal{R}}_{\lambda \mu}^{\tau}(t)=\int d(\mathbf{p}, \mathbf{r})\{r \otimes r\}_{\lambda \mu} \delta \kappa_{e}^{\tau i}(\mathbf{r}, \mathbf{p}, t), \\
& \tilde{\mathcal{P}}_{\lambda \mu}^{\tau}(t)=\int d(\mathbf{p}, \mathbf{r})\{p \otimes p\}_{\lambda \mu} \delta \kappa_{e}^{\tau i}(\mathbf{r}, \mathbf{p}, t),
\end{aligned}
$$

where $\varsigma=\uparrow \uparrow, \downarrow \downarrow, \uparrow \downarrow, \downarrow \uparrow$, indexes $o$ and $e$ mean "time odd" and "time even", $\quad\{r \otimes r\}_{\lambda \mu}=\sum_{\sigma, v} C_{1 \sigma, 1 v}^{\lambda \mu} r_{\sigma} r_{v}$. Following Ref. [9] we neglect the real part of the variation of the anomalous density $\delta \kappa^{r}$, because $\delta \kappa^{r}(\mathbf{r}, \mathbf{p}) \ll \delta \kappa^{i}(\mathbf{r}, \mathbf{p})$. Further, we neglect the diagonal in spin matrix elements $\kappa^{s s}$. It is shown in [12] that this approximation works very well for the monopole pairing considered here. Symmetry properties of the matrices $\hat{\kappa}$ allow one to replace the functions $\kappa^{\downarrow \uparrow}(\mathbf{r}, \mathbf{p})$ with the functions $\bar{\kappa}^{\uparrow \downarrow}(\mathbf{r}, \mathbf{p})$. Finally, the notation $\kappa \equiv \kappa^{\uparrow \downarrow}$ is introduced.

We are interested in the scissors mode with quantum number $K^{\pi}=1^{+}$. Therefore, we only need the part of dynamic equations with $\mu=1$.

\section{Hidden angular momenta}

This phenomenon is provided by the spin of nucleons and naturally has the essential influence on all nuclear properties connected with the spin. Let us analyze the linearization procedure of the equations of motion. We consider small deviations of the system from the equilibrium, so all variables are written as a sum of their equilibrium value plus a small deviation:

$L_{\lambda \mu}^{\tau S}(t)=L_{\lambda \mu}^{\tau S}(\mathrm{eq})+\mathcal{L}_{\lambda \mu}^{\tau S}(t), R_{\lambda \mu}^{\tau S}(t)=R_{\lambda \mu}^{\tau S}(\mathrm{eq})+\mathcal{R}_{\lambda \mu}^{\tau S}(t)$, et al.

Linearized equations for deviations depend on the equilibrium values $R_{\lambda \mu}^{\tau \varsigma}(\mathrm{eq})$ and $L_{\lambda \mu}^{\tau \varsigma}(\mathrm{eq})$, which are the input data of the problem. It is easy to show [12] that

$$
L_{10}^{\uparrow \uparrow}(\mathrm{eq})=-L_{10}^{\downarrow \downarrow}(\mathrm{eq}) \neq 0 .
$$

So the ground state of a nucleus consists of two equal parts with nonzero angular momenta with opposite directions (counter rotation), which compensate each other resulting in the zero total $\left(L_{10}^{\uparrow \uparrow}(\mathrm{eq})+L_{10}^{\downarrow \downarrow}(\mathrm{eq})\right)$ angular momentum (hidden angular momenta). Being not pronounced in the ground state, this phenomenon can become apparent in the excited states. It is obvious, for example, that it should influence essentially the excitation of the spin scissors mode.

\section{Results of calculations}

Imposing the time evolution via $\mathrm{e}^{i E t / \hbar}$ for all variables (18) one transforms a set of dynamic equations into a set of algebraic equations. It contains 23 equations with $\mu=1$. We find seven roots with exactly $E=0$ and 16 roots which are non zero. In this paper we consider only the two lowest roots corresponding to the orbital and spin scissors. The qualitative picture of high-lying modes remains largely unchanged in comparison with [11]. Seven integrals of motion corresponding to Goldstone modes (zero roots) can be found analytically [12]. 

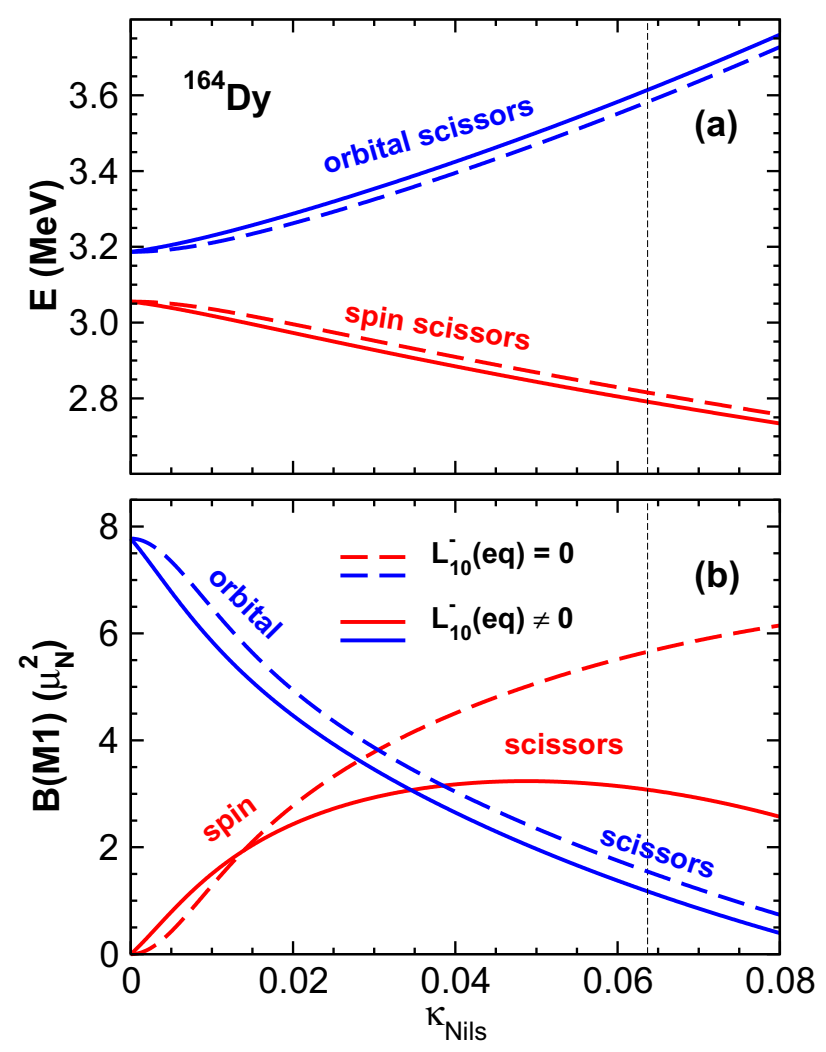

Figure 2. (Color online) The energies $E$ (a) and $B(M 1)$-factors (b) as a functions of the spin-orbital strength constant $\kappa_{\text {Nils }}$. The dashed lines - calculations with $L_{10}^{-}(\mathrm{eq})=0$, the solid lines $L_{10}^{-}(\mathrm{eq}) \neq 0$. Pair correlations and spin-spin forces are included.

Our calculations without pairing [11] have shown that the results are strongly dependent on the values of the strength constants of the spin-spin interaction. How sensitive are they to the strength of the spin-orbital potential $\eta$ ? The results for ${ }^{164} \mathrm{Dy}$ are shown in Fig. 2 as a function of $\kappa_{\mathrm{Nils}}=\eta /\left(2 \hbar \omega_{0}\right)$. One observes a rather strong dependence of the results on the value of $\kappa_{\text {Nils }}$ : the splitting $\Delta E$ and $M 1$ strength of the spin scissors grow with increasing $\kappa_{\text {Nils }}$ whereas the $B(M 1)$ value of the orbital scissors decreases. The main role of the spin-orbital potential consists in the excitation of the spin scissors mode. This mode is not excited directly by the electromagnetic field. It can be excited only indirectly, via the coupling with the conventional (orbital) scissors mode by means of the spinorbital interaction [11]. As a result, the $B(M 1)$ value of the spin scissors becomes zero in the case $\kappa_{\text {Nils }}=0$. The best value of $\kappa_{\text {Nils }}$ for rare earth nuclei was established [13] to be 0.0637 (it is marked by the dotted vertical line in Fig. 2).

We made the calculations taking into account the nonzero value of $L_{10}^{-}(\mathrm{eq})=L_{10}^{\uparrow \uparrow}(\mathrm{eq})-\mathrm{L}_{10}^{\downarrow \downarrow}(\mathrm{eq})$. It is extracted from the standard Nilsson scheme [13] and $L_{10}^{-}(\mathrm{eq})=i 21.22$ for ${ }^{164} \mathrm{Dy}$. Figure 2 demonstrates the strong influence of the hidden angular momenta on the spin scissors mode whose $B(M 1)$ value is strongly decreased (for $\kappa_{\mathrm{Nils}}>0.02$ ) after including $L_{10}^{-}(\mathrm{eq})$. The $B(M 1)$ value of the orbital scissors also reduces, but not
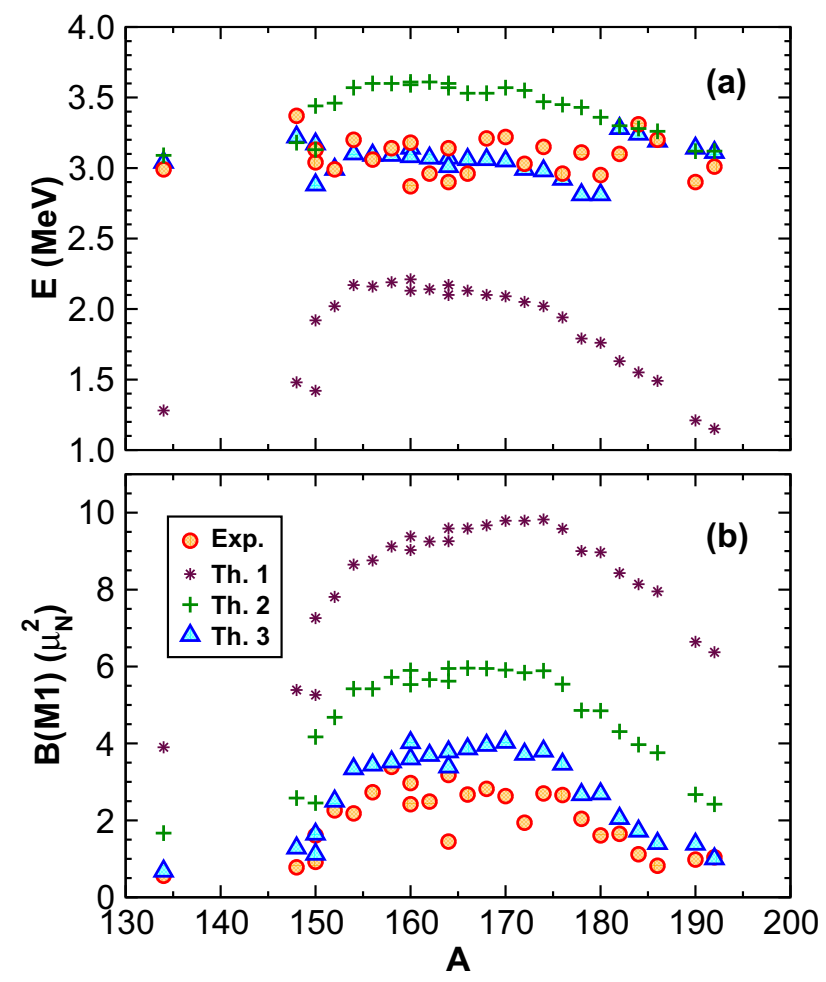

Figure 3. (Color online) The energies $E$ (a) and $B(M 1)$-factors (b) as functions of the mass number A. The experimental values of $E$ and $B(M 1)$ are taken from [18] and references therein. Th.1 - the calculations without spin degrees of freedom and pair correlations, Th. 2 - only pairing is included, Th. 3 - pairing and spin degrees of freedom are taken into account.

so much, and the reduction being practically independent on $\kappa_{\text {Nils }}$. The influence of $L_{10}^{-}(\mathrm{eq})$ on the energies of both scissors is negligible, leading to a small increase of their splitting.

The results of systematic calculations for rare-earth nuclei are shown in Fig. 3. The comparison of the results with (Th.2) and without (Th.1) pairing allows one to understand the extremely important role of pair correlations in the description of the scissors mode properties. It is seen also (Th.3) that only introducing into consideration the spin degrees of freedom (and not forgetting about $L_{10}^{-}(\mathrm{eq}) \neq 0$ term) allows one to obtain a satisfactory agreement with experimental data on energies and excitation probabilities.

For the group of well deformed nuclei with deformations $\delta \geq 0.18$ all presented results were obtained without any fitting with $\kappa_{\mathrm{Nils}}=0.0637$ and with the value of the pairing interaction constant $V_{0}=25 \mathrm{MeV}$. For the weakly deformed nuclei with $0.14 \leq \delta \leq 0.17$ reasonable results are obtained with $\kappa_{\text {Nils }}=0.05$ and $V_{0}=27 \mathrm{MeV}$.

\section{Conclusion}

The WFM method is generalized to take into account spin degrees of freedom and pair correlations simultaneously.

The inclusion of the spin into the theory allow us to discover several new phenomena. One of them, the nu- 
clear spin scissors, was described and studied in [10, 11], where some indications on the experimental confirmation of its existence in actinides were discussed. Another phenomenon, the hidden angular momenta, is described in [12]. This phenomenon has great influence on the excitation probability of the spin scissors mode. On the other hand, the $B(M 1)$ values of spin scissors and the energies of both, spin and orbital, scissors are very sensitive to pair correlations. As a result, these two factors, the hidden angular momenta and pairing, working together, improve substantially the agreement between the theory and experiment.

\section{References}

[1] R. R. Hilton, Talk presented at the International Conference on Nuclear Structure (Joint Institute for Nuclear Research, Dubna, Russia, 1976) (unpublished).

[2] R.R. Hilton, Ann. Phys. (NY) 214, 258 (1992)

[3] T. Suzuki and D.J. Rowe, Nucl. Phys. A 289, 461 (1977)

[4] N. Lo Iudice and F. Palumbo, Phys. Rev. Lett. 41, 1532 (1978)

[5] D. Bohle, A. Richter, W. Steffen, A.E.L. Dieperink, N. Lo Iudice, F. Palumbo, and O. Scholten, Phys. Lett. B. 137, 27 (1984)

[6] D. Zawischa, J. Phys. G: Nucl. Part. Phys. 24, 683 (1998)
[7] J. Enders, P. von Neumann-Cosel, C. Rangacharyulu, and A. Richter, Phys. Rev. C 71, 014306 (2005)

[8] V.G. Soloviev, A.V. Sushkov, N.Yu. Shirikova, Phys. Part. Nucl. 31, 385 (2000)

[9] E.B. Balbutsev, L.A. Malov, P. Schuck, and M. Urban, Phys. At. Nucl. 72, 1305 (2009)

[10] E.B. Balbutsev, I.V. Molodtsova, and P. Schuck, Nucl. Phys. A 872, 42 (2011)

[11] E.B. Balbutsev, I.V. Molodtsova, and P. Schuck, Phys. Rev. C 88, 014306 (2013)

[12] E.B. Balbutsev, I.V. Molodtsova, and P. Schuck, Phys. Rev. C 91, 064312 (2015)

[13] P. Ring and P. Schuck, The Nuclear Many-Body Problem (Springer, Berlin, 1980)

[14] D.A. Varshalovitch, A.N. Moskalev, and V.K. Khersonski, Quantum Theory of Angular Momentum (World Scientific, Singapore, 1988)

[15] A. Bohr and B. Mottelson, Nuclear Structure, Vol. 2 (Benjamin, New York, 1975)

[16] E.B. Balbutsev, P. Schuck, Ann. Phys. 322, 489 (2007)

[17] P. Sarriguren, E. Moya de Guerra, and R. Nojarov, Z. Phys. A 357, 143 (1997)

[18] N. Pietralla, P. von Brentano, R.-D. Herzberg, U. Kneissl, N. Lo Iudice, H. Maser, H.H. Pitz, and A. Zilges, Phys. Rev. C 58, 184 (1998) 